\title{
Single Dose Activated Charcoal
}

To the Editor:

The study by LoVecchio et al [1] in the September issue of Journal of Medical Toxicology addresses the feasibility of administration of single-dose activated charcoal (SDAC) with respect to current practice guidelines, referencing the practice guidelines of the American Academy of Clinical Toxicology (AACT) and European Association of Poison Centres and Clinical Toxicologists (EAPCCT) [2].

LoVecchio et al [1] also reference a review [3] to support the statement "Although undetermined, the benefits of administering AC within the first hour of ingestion outweigh the risk of adverse events." However, this review states "At present we are unable to demonstrate benefit from the administration of SDAC. The risks include death, long-term pulmonary disease, charcoal peritoneum, and corneal abrasion. The benefits of a treatment must be clearly demonstrated if one of the risks of the procedure is death. We must reassess if the harm caused by the administration of SDAC is greater than the harm caused by the poisoning. If this is the case, and benefit cannot be demonstrated, the practice of administering SDAC should be abandoned in the mild to moderately poisoned patient [3]."

The 1997 SDAC Position Statement (PS) (published by AACT and EAPCCT) states "SDAC should not be administered routinely in the management of poisoned patients. Based on volunteer studies, the effectiveness of AC decreases with time; the greatest benefit is within one hour of ingestion. The administration of AC may be considered if a patient has ingested a potentially toxic amount of poison (which is known to be adsorbed by charcoal) up to one hour previously; there are insufficient data to support or exclude its use after 1 hour of ingestion. There is no evidence that the administration of AC improves clinical outcome [2]."

This PS was revised in 2005 by the same Societies and the revision states "A review of the literature since the preparation of the 1997 SDAC Position Statement revealed no new evidence that would require a revision of the conclusions of the Statement[4]."

The two Societies carefully worded the PS to ensure clarity of meaning. Neither the 1997 nor the 2005 PS recommended the routine administration of AC within one hour of ingestion. Yet many of our toxicology publications continue to quote them as doing so.

How reviews, studies, and practice guidelines are interpreted is not based on data alone and depends on interpretation of the evidence and on clinicians' experience [5]. Interpreting the literature involves value judgments. Because many of our clinical toxicology publications continue to quote the PS as recommending $\mathrm{AC}$ within an hour of ingestion, a practice continues based on a recommendation that does not exist.

When there is little or no evidence, we must make clinical recommendations in the light of available evidence and clinical experience. We have to carefully assess which of these we are using as a basis for our recommendations.

Donna Seger, MD

Medical Director

TN Poison Center

Associate Professor of Medicine and Emergency Medicine

Vanderbilt University Medical Center

\section{REFERENCES}

1. LoVecchio F, Shriki J, Innes K, Bermudez J. Journal of Medical Toxicology. 2007;3(3)100-102.

2. Chyka PA, Seger D. Position statement; single dose activated charcoal. American Academy of Clinical Toxicology; European Association of Poisons Centres and Clinical Toxicologists. J Toxicol Clin Toxicol. 1997;35(7);721-24.

3. Seger D, Single-dose activated charcoal-backup and reassess. J Toxicol Clin Toxicol. 2004;42(1);101-110.

4. AACT;EAPCCT. Position Paper; Single-Dose Activated Charcoal. Clinical Toxicology. 2005; 2, 51-87.

5. Raine R, Sanderson C, Hutchings A, Carter S, Larkin, K, Black N. An experimental study of determinants of group judgments in clinical guideline development. Lancet. 2004; 364:429-37. 